\title{
LA INFORMACIÓN NO FINANCIERA Y EL DESEMPEÑO FINANCIERO EMPRESARIAL*
}

Recibido: 06 de octubre de 2017 - Aprobado: 19 de febrero de 2018

DOI: $10.22395 /$ seec.v21n46a8

\author{
Diego Andrés Correa Mejía** \\ Diana Camila Reyes Naranjo*** \\ Keila Marley Montoya Beltrán ${ }^{* * *}$
}

\section{RESUMEN}

Este artículo pretende validar los efectos que tienen los reportes de sostenibilidad en el desempeño financiero de las empresas colombianas. A través de un modelo de datos de panel y la información de 30 empresas que cotizan en la bolsa de valores colombiana para el periodo 2012-2015, se evalúa la incidencia que tienen los reportes de sostenibilidad sobre el desempeño financiero de este grupo de empresas. Se concluye que el tamaño de la empresa y la cantidad de indicadores ambientales tienen un efecto positivo en el desempeño financiero, mientras que la antigüedad de la misma causa lo contrario.

\section{PALABRAS CLAVE}

Valor de las empresas; reportes de sostenibilidad; desempeño financiero; responsabilidad social empresarial; Colombia.

\section{CLASIFICACIÓN JEL}

G32, G31

\section{CONTENIDO}

Introducción; 1. Algunos antecedentes sobre los reportes de sostenibilidad y el desempeño financiero de las empresas; 2 . Estrategia metodológica; 3 . Algunos resultados obtenidos; 4. Bibliografía.

Este artículo de reflexión, elaborado en 2017, surge con el objetivo de evaluar los efectos que han tenido los informes de sostenibilidad en el desempeño financiero de las empresas en el contexto colombiano durante el periodo 2012-2015.

* Contador público, Universidad de Antioquia, Medellín, Colombia. Profesor e investigador, Facultad de Ciencias Económicas, Universidad de Antioquia. Aspirante al título de magíster en finanzas, Universidad de Antioquia. Dirección: calle 67 n. ${ }^{\circ}$ 53-108, bloque 13, oficina 116, Medellín, Colombia. Correo electrónico: diegoa.correa@udea.edu.co

** Contadora pública, Universidad de Antioquia (Medellín, Colombia). Asistente de auditoría, Deloitte $\mathcal{E}$ Touche Ltda. Dirección: calle 67 n.53-108, Medellín, Colombia. Correo electrónico: camila.reyes@ udea.edu.co

${ }^{* * *}$ Estudiante de Contaduría Pública, Universidad de Antioquia (Medellín, Colombia). Dirección: calle 67 n.53-108, Medellín, Colombia. Correo electrónico: keila.montoya@udea.edu.co 


\title{
NON-FINANCIAL INFORMATION AND BUSINESS FINANCIAL PERFORMANCE
}

\begin{abstract}
This paper aims to validate the effects that sustainability reports have on the financial performance of Colombian companies. Through a panel data model and the information of 30 companies listed on the Colombian stock exchange for the 2012-2015 period, the impact of sustainability reports on the financial performance of this group of companies is evaluated. It is concluded that the size of the company and the number of environmental indicators have a positive effect on financial performance, while its antiquity causes the opposite.
\end{abstract}

\section{KEYWORDS}

Value of the companies; sustainability reports; financial performance; corporate social responsibility; Colombia.

\section{JEL CLASSIFICATION}

G32, G31

\section{CONTENT}

Introduction; 1. Some Background on the Companies' Sustainability Reports and Financial Performance; 2. Methodological Strategy; 3. Some Results Achieved; 4. Bibliography.

\section{A INFORMAÇÃO NÃO FINANCEIRA E 0 DESEMPENHO FINANCEIRO EMPRE- SARIAL \\ RESUMO}

Este artigo pretende validar os efeitos que os relatórios de sustentabilidade têm no desempenho financeiro das empresas colombianas. Através de um modelo de dados em painel e da informação de 30 empresas que cotizam na bolsa de valores colombiana para o período de 2012-2015, avalia-se a incidência que os relatórios de sustentabilidade têm sobre o desempenho financeiro desse grupo de empresas. Conclui-se que o tamanho da empresa e a quantidade de indicadores ambientais têm um efeito positivo no desempenho financeiro, enquanto a antiguidade da empresa apresenta um efeito contrário.

\section{PALAVRAS-CHAVE}

Valor das empresas; relatórios de sustentabilidade; desempenho financeiro; responsabilidade social empresarial; Colômbia.

\section{CLASSIFICAÇÃO JEL}

G32, G31

\section{CONTEÚDO}

Introdução; 1. Alguns antecedentes sobre os relatórios de sustentabilidade e o desempenho financeiro das empresas; 2. Estratégia metodológica; 3. Alguns resultados obtidos: 4. Bibliografia. 


\section{INTRODUCCIÓN}

Las empresas han identificado que no es suficiente presentar solo información financiera: las dinámicas del mercado y las demandas sociales exigen mucho más. Los informes de sostenibilidad han surgido por ello como un medio para presentar información no financiera, que permita reflejar una imagen transparente e impulsar una buena reputación. Ferrero (2013) manifiesta que la estrategia sostenible ayuda a la creación de imagen y reputación favorable ante los inversionistas, consumidores y agentes del mercado en general, y por ende, puede repercutir en mejores niveles de rentabilidad corporativa.

Con el objetivo de identificar los efectos de los reportes de sostenibilidad en el desempeño financiero de las empresas colombianas, para el ejercicio presentado en este artículo se tomó una muestra de 30 empresas que operan en el territorio colombiano, y se analizó la información presentada bajo la guía Global Reporting Initative (GRI) para el período 2012-2015 mediante una metodología de datos de panel. En el análisis realizado se encontró que el tamaño de la empresa y la cantidad de indicadores ambientales tienen un efecto positivo en el desempeño financiero; mientras que la antigüedad de esta acarrea lo contrario. El estudio aborda las posiciones teóricas que han establecido diferentes autores sobre la relación entre los reportes de sostenibilidad y el desempeño financiero de las empresas. Esto se complementa con un análisis de resultados basado en la muestra y metodología establecidas, que permite generar las conclusiones.

\section{ALGUNOS ANTECEDENTES SOBRE LOS REPORTES DE SOSTENIBILIDAD Y EL DESEMPEÑO FINANCIERO DE LAS EMPRESAS}

\subsection{Responsabilidad social empresarial (RSE) y los efectos en las empresas}

A finales del siglo XX, las organizaciones empresariales realizaban actividades de solidaridad social sin tener un referente frente a lo que hoy se denomina responsabilidad social empresarial (RSE). El concepto ha evolucionado desde entonces: las empresas han tomado más consciencia sobre lo que implica este concepto y, con esto, se comenzaron a adoptar prácticas concretas en beneficio de la sociedad. Se pasó, entonces, a una situación en la que empresas, sociedad y Estado perseguirían un mismo objetivo, como lo señala Correa (2007).

Según Correa (2007), los factores de más incidencia en la evolución del concepto de RSE son la globalización; la liberalización del comercio y sus reformas regulatorias; el desarrollo ambiental sostenible; los códigos de buen gobierno; la acelerada degradación del medio ambiente; y la exclusión de sectores de la sociedad 
como resultado del desarrollo, entre otros. La RSE cobra cada vez mayor fuerza, y las empresas toman más consciencia de los beneficios que estas prácticas le generan. Según el estudio realizado por Alonso-Almeida et al. (2012), la RSE favorece la confianza y el sentido de pertenencia de los empleados, lo cual permite que los trabajadores tengan una mejor disposición hacia el trabajo, al tiempo que disminuya la rotación de personal y, por consiguiente, los costos de reclutamiento. Asimismo, como lo plantean Kim, Li y Li (2014), con la RSE se beneficia la calidad y, con ella, la satisfacción de los clientes. El argumento anterior es apoyado por Borjas (2007): este afirma que la imagen de la empresa mejora en los ámbitos local e internacional gracias a la RSE, lo que favorece la reputación frente a mercados comerciales y financieros.

Autores como El Ghoul et al. (2011), muestran que el costo de capital social es más bajo para empresas con altas prácticas de RSE; y muestran que los inversionistas prefieren compañías con estas prácticas al momento de invertir. Este argumento es apoyado por Blazenko (1987), quien manifiesta que el costo de capital se reduce cuando la información sobre activos reales es simétrica: los gerentes tendrían mayor preferencia al momento de financiarse mediante el patrimonio y no por deuda, siempre y cuando esta tenga simetría.

También se ha manifestado la incidencia que tienen las prácticas de RSE en la creación de valor de las compañías. A través del estudio realizado por Fatemi, Fooladi y Tehranian (2015) se demuestra que los gastos de RSE crean valor para la firma. Para confirmar lo dicho, estos autores realizaron simulaciones por medio de las cuales es posible comprobar que la RSE se convierte en una forma de creación de valor.

Como lo afirman Alonso et al. (2016), la información no financiera ha logrado tener mayor visibilidad porque, además de dar cuenta de la generación de recursos financieros, las empresas también son conscientes de la importancia de mostrar cómo se generan estos recursos, qué recursos naturales se requieren y qué afectaciones tiene para la sociedad que participa de forma directa e indirecta en los procesos productivos de la empresas.

Los informes que presentan las empresas sobre su desempeño financiero ayudan a los inversionistas a tener mayor confiabilidad. No obstante surge la necesidad de complementar dichos informes en términos cuantitativos y cualitativos, a fin de que no se vea limitada la información y se logre reducir el costo de capital, como lo plantean Fatemi, Fooladi y Tehranian (2015), lo que a su vez reduce el riesgo de la información (Plumlee et al.,2015).

En este sentido, los reportes de sostenibilidad se han convertido en el medio de comunicación con el que las empresas generan información no financiera para 
los grupos de interés sobre las acciones de RSE. Estos buscan informar a los grupos sobre la forma como las empresas suplen sus necesidades sin afectar de forma negativa su entorno, ni dejar a un lado sus propias necesidades del futuro, como lo mencionan autores como Almeida, Marimon, y Llach (2015). En las investigaciones sobre las adopciones de estándares de reportes organizacionales, Marimon et al. (2012) señalan que los países con mayor desarrollo económico han adoptado con más facilidad las prácticas exigidas en las guías de sostenibilidad.

A partir de una revisión de la literatura empírica y un metanálisis, Fifka (2013) muestra que los reportes de sostenibilidad realizados en los países desarrollados han sido acogidos en mayor medida y con más rigurosidad en comparación con los países en vía de desarrollo. En el estudio se verifica que se ha prestado poca atención a la transmisión de los reportes de sostenibilidad en Latinoamérica.

Nuñez (2003), por su parte, manifiesta que el hecho de incluir el concepto estudiado en los informes elaborados por las empresas marca una tendencia que contribuye a mejorar su desempeño económico, a la vez que aumenta su competitividad y, por ende, su valor. Ahora bien, mientras que Callan y Thomas (2009) hallaron una relación positiva entre la RSE y el desempeño financiero, Nelling y Webb (2009), luego de llevar a cabo un estudio con el cual buscaban constatar la relación de estos dos conceptos, concluyeron que existe una relación débil entre la RSE y el desempeño financiero.

\subsection{Estándares utilizados para la presentación de la RSE}

Como consecuencia de la evolución que ha tenido el concepto de RSE, se han creado diversos estándares como medio para el reporte de esta información por parte de las empresas, entre los que se encuentra la ISO 26000. El objetivo de dicho estándar (de aplicación voluntaria y no certificable) es generar orientación a las compañías sobre responsabilidad social, y puede llegar a implementarse como parte de las actividades de la política pública. Su función radica en ayudar a las organizaciones a contribuir al desarrollo sostenible.

La Norma SA 8000, expedida por Social Accountability International (2001), a su turno, específica los requisitos de responsabilidad social que permiten a una compañía desarrollar sus operaciones de manera acorde con los principios de RSE señalados en la norma, con el objeto de manejar aquellos asuntos que están bajo su control o influencia. Asimismo, permite demostrar a las partes interesadas que las prácticas implementadas por las firmas están acordes a los requerimientos de RSE impuestos por la misma norma. 
En Brasil, el Instituto Ethos ha desarrollado un sistema de indicadores que permite a las firmas acompañar las acciones de responsabilidad social de sus proveedores y de sus clientes, como lo señala Viteri (2010). Así entonces, los llamados indicadores Ethos pretenden facilitar la gestión de la RSE, en tanto buscan involucrar a los agentes de la cadena de valor de las firmas.

Por su parte, el GRI ha elaborado un marco que aporta directrices sobre la elaboración de memorias de RSE, incluyendo los impactos económicos, sociales y ambientales de una empresa de acuerdo con Duque, Cardona y Rendón (2013)

Rodriguez y Rios (2016) señalan que la presentación de la información bajo el GRI es relevante para las organizaciones y grupos de interés, permitiendo que la información sea vista de manera confiable bajo los aspectos sociales, ambientales y económicos

Los reportes de sostenibilidad han sido utilizados por las empresas con distintas finalidades, según el sector social y económico en el que desarrollen su objeto social. Como lo mencionan Gómez y Quintanilla (2012), los estándares para la emisión de los reportes tienen como propósito plantear marcos para informes sociales: buscan normalizar patrones comunes y establecerlos en una guía, cuyo fin es garantizar y cuidar los recursos dirigidos a una dinámica económica equilibrada. Los reportes de sostenibilidad se han convertido, entonces, en un mecanismo de comunicación práctico para el diálogo con los grupos de interés (Willis, 2003).

Para el desarrollo del presente estudio se tomó como referencia la guía propuesta por el GRI en sus versiones G3.1 y G4, en la medida en que es útil para estandarizar la información que se presenta en los informes de sostenibilidad, en busca que pueda ser comparable entre empresas y entendible para los grupos de interés. De igual manera, en el contexto colombiano el GRI es el estándar con mayor aplicación por parte de las empresas. Para el propósito del trabajo, el cambio de la guía no tuvo ningún impacto en la recolección de la información.

\section{ESTRATEGIA METODOLÓGICA}

En esta sección se mostrará en detalle la forma como se seleccionaron las empresas que componen la muestra del estudio, al tiempo que se realizará una breve caracterización sobre los sectores que se encuentran representados y la cantidad de observaciones que se tienen de las empresas en el período comprendido entre 2012-2015. Luego, se describirán las variables explicativas del desempeño financiero y las variables de control que se tuvieron en cuenta para la formulación del modelo de datos de panel. Por último, se planteará de manera formal el modelo econométrico desarrollado para obtener los resultados. 


\subsection{Empresas incluidas en el estudio}

El desarrollo del estudio se centró en las empresas que cotizan en la bolsa de valores y presentaron información con la metodología de GRI en el periodo 2012-2015, fundamentado en el análisis realizado por Fifka (2013). La muestra está compuesta por 30 empresas que cumplen las características mencionadas, que además se encuentran establecidas en el territorio colombiano (la lista de las empresas incluidas se puede consultar en el anexo), de las cuales se obtuvo información acerca de sus reportes de sostenibilidad. La composición de la muestra por sector económico se detalla en la tabla 1, según el sector establecido en el software Economatica.

Tabla 1. Distribución de las empresas por sector económico

\begin{tabular}{|l|c|c|}
\hline \multicolumn{1}{|c|}{ Sector } & Cantidad & Frecuencia \\
\hline Aeronáutico & 1 & $3,3 \%$ \\
\hline Energía & 7 & $23,3 \%$ \\
\hline Cementero & 3 & $10,0 \%$ \\
\hline Minería & 5 & $16,7 \%$ \\
\hline Telecomunicaciones & 3 & $10,0 \%$ \\
\hline Comercial & 3 & $10,0 \%$ \\
\hline Industrial & 4 & $13,3 \%$ \\
\hline Salud & 1 & $3,3 \%$ \\
\hline Construcción & 1 & $3,3 \%$ \\
\hline Infraestructura & 1 & $3,3 \%$ \\
\hline Transporte & 30 & $3,3 \%$ \\
\hline Total & & $100,0 \%$ \\
\hline
\end{tabular}

Fuente: elaboración propia.

Se destaca la participación de las empresas de los sectores de energía, cementero, minería, telecomunicaciones, comercial e industrial, los cuales representan el 83,3 \% del total de las empresas estudiadas. Debido a que el estudio se realizó a lo largo de 4 años, se obtuvieron 94 observaciones por empresa y por año (de las 30 estudiadas), ya que algunas no presentaron la información de manera consecutiva. La tabla 2 muestra la composición de las observaciones empresa-año obtenidas por sector. 
Diego Andrés Correa Mejía - Diana Camila Reyes Naranjo - Keila Marley Montoya Beltrán

Tabla 2. Observaciones por empresa, año y sector

\begin{tabular}{|l|c|c|}
\hline \multicolumn{1}{|c|}{ Sector } & Cantidad & Frecuencia \\
\hline Aeronáutico & 4 & $4,3 \%$ \\
\hline Energía & 23 & $24,5 \%$ \\
\hline Cementero & 8 & $8,5 \%$ \\
\hline Minería & 14 & $14,9 \%$ \\
\hline Telecomunicaciones & 12 & $12,8 \%$ \\
\hline Comercial & 12 & $12,8 \%$ \\
\hline Industrial & 14 & $14,9 \%$ \\
\hline Salud & 3 & $3,2 \%$ \\
\hline Construcción & 1 & $1,1 \%$ \\
\hline Infraestructura & 1 & $1,1 \%$ \\
\hline Transporte & 2 & $2,1 \%$ \\
\hline Total & 94 & $100,0 \%$ \\
\hline
\end{tabular}

Fuente: elaboración propia.

Se consultó la información presentada en los informes sostenibilidad que cada empresa publica en su respectiva página web. Lo anterior representa una limitación de acceso a la información, ya que algunas empresas no publican los reportes en dichos espacios.

\subsection{Variables}

\subsubsection{Variable dependiente}

Según Montoro y Navarro (2010), la Q de Tobin es una variable financiera que refleja el valor que tienen la relación del valor de mercado y el valor en libros de las acciones de la compañía. Se espera que esta variable sea afectada de forma positiva por las variables seleccionadas a continuación y que dicha afectación o influencia sea significativa, de acuerdo con lo establecido por Garay y González (2005).

La Q de Tobin se utiliza con frecuencia para probar la relación que existe entre los informes de responsabilidad social y el desempeño financiero, en tanto representa, según lo expuesto por Luo y Bhattacharya (2006) y Wang y Qian (2011), los beneficios a largo plazo de las firmas. 


\subsubsection{Variables Independientes}

Cantidad de mujeres en la empresa (igualdad): según Godoy y Mladinic (2009), la feminización de la fuerza laboral constituye uno de los fenómenos más relevantes ocurridos en los mercados laborales en las últimas décadas; se ha incrementado la presencia de las mujeres en diferentes cargos, y su labor aporta beneficios a las empresas. Las mujeres han tenido gran incidencia cuando se encuentran en cargos directivos: por regla general, al encontrarse en esa situación tienden a alentar la participación y generan valor a la comunicación interpersonal (Pérez, 2001).

Alonso et al. (2016), señalan que las empresas que lleven a cabo una política de igualdad, en el desarrollo de la cual se contraten hombres y mujeres, actuarán conforme a las demandas sociales, lo que puede incidir en la aceptación y reputación en el sector. Se espera que, cuanto mayor sea el equilibrio entre la cantidad de hombres y mujeres que laboran en una empresa, mayor será su efecto en el desempeño financiero de la misma.

Miembros independientes de las juntas directivas: estos generan transparencia e imparcialidad en las decisiones tomadas en la empresa. Al respecto, autores como Acero y Alcalde (2012) afirman que las juntas directivas pequeñas con buena participación de miembros independientes generarán un efecto positivo en términos de rentabilidad, al considerar que con dicha estructura organizativa la junta realizará de una forma más eficiente sus labores. Así entonces, se prevé un efecto positivo en el desempeño financiero de las empresas cuando su junta directiva está conformada por un número representativo de miembros independientes.

Indicadores reportados en la información ambiental: para la elaboración del GRI son necesarios unos protocolos de indicadores de desempeño, entre ellos los ambientales. Según Moneva y Ortas (2009), las firmas con un nivel de desempeño ambiental adecuado cuentan con mejores resultados financieros, por lo cual se espera de ellos una relación positiva frente al desempeño financiero de la compañía.

Aseguramiento del reporte: el compromiso de aseguramiento se da cuando se pone a disposición el informe de sostenibilidad ante un tercero independiente, con el fin de que este emita un juicio sobre la veracidad de la información reportada. Saenz et al. (2014) afirman que el aseguramiento mejora el grado de confianza de los usuarios de los reportes. Si bien no es obligatoria, se considera que incorporar esta variable en los reportes tiene un efecto positivo en el desempeño financiero de las firmas; según García, Zamora y Ruiz (2009), el aseguramiento de la información no financiera aumenta el nivel de transparencia de las compañías. 


\subsubsection{Variables de control}

Nivel de endeudamiento: relación existente entre el total de pasivos y el total de activos, e indica el porcentaje de los activos que se han conseguido con financiamiento de terceros. De acuerdo con Cuervo y Rivero (1986), el endeudamiento es un factor generador o reductor de la rentabilidad de los fondos propios. Un nivel de endeudamiento adecuado permite maximizar tanto el valor de la empresa como el desempeño financiero. No obstante, un endeudamiento excesivo puede tener efectos negativos sobre el desempeño financiero debido a los altos gastos financieros que puede acarrear, los cuales afectan las utilidades de las firmas (Alonso-Almeida et al., 2012).

Tamaño de la empresa: cantidad de activos de los que dispone una empresa. En términos generales, Alonso et al. (2016) establecen que existe una relación positiva entre el tamaño de la organización, las actividades de RSE y, por ende, el desempeño financiero. Según lo estudiado por Preston y O'Bannon (1997), las empresas de mayor tamaño tienden a contar con un mejor desempeño financiero.

Antigüedad o edad de la organización: edad o medida en número de años transcurridos desde el nacimiento de la organización (Alonso et al., 2016). Según los trabajos realizados por Choi (1999) y Hamid (2004), existe una relación positiva entre la edad de la organización y el desempeño financiero, ya que las empresas maduras y establecidas en el mercado cuentan con procesos estandarizados, los cuales les confieren más probabilidades de tener un mejor desempeño financiero. Sin embargo, según Pozniak et al. (2011), el hecho de que las empresas antiguas puedan tener un nivel elevado de activos podría influir de manera negativa en su desempeño financiero; esto por cuanto las utilidades de las firmas no crecen en la misma proporción que los activos o el patrimonio, lo cual afecta indicadores como el ROA (rentabilidad del activo) y el ROE (rentabilidad del patrimonio).

\subsection{Modelo}

Se desarrolló un modelo de datos panel, por medio del cual se pueden identificar los efectos de las variables independientes en la variable dependiente, teniendo en cuenta los efectos temporales y estructurales. Con base en la información encontrada en los reportes de sostenibilidad de las empresas seleccionadas se realizó un análisis de regresión, con el fin de identificar cuáles de las variables seleccionadas afectan de formas positiva, negativa o no significativa el desempeño financiero de las mismas. Como variable dependiente, la $\mathrm{Q}$ de Tobin representa el desempeño financiero de la empresa. La selección de las variables que pueden influir en el desempeño financiero se basó en una revisión bibliográfica precedente. Las variables identificadas como independientes del modelo se muestran en la tabla 3. 
La información no financiera y el desempeño financiero empresarial

Tabla 3. Variables que inciden en el desempeño financiero y relación esperada

\begin{tabular}{|c|c|c|c|}
\hline Variable & Medición & $\begin{array}{l}\text { Efecto } \\
\text { esperado }\end{array}$ & Autor \\
\hline Igualdad & $\begin{array}{l}\text { Porcentaje de mujeres que } \\
\text { trabajan en la compañía. }\end{array}$ & Positivo & $\begin{array}{l}\text { Godoy y Mladinic (2009), } \\
\text { Pérez (2001) Alonso et al. } \\
\text { (2016) }\end{array}$ \\
\hline Aseguramiento del reporte & $\begin{array}{l}0=\text { no está asegurado. } \\
1=\text { está asegurado. }\end{array}$ & Positivo & $\begin{array}{l}\text { García, Zamora y Ruiz } \\
\text { (2009), Alonso J. et al. } \\
\text { (2016) }\end{array}$ \\
\hline $\begin{array}{l}\text { Cantidad de miembros in- } \\
\text { dependientes }\end{array}$ & $\begin{array}{l}\text { Cantidad de miembros in- } \\
\text { dependientes en la junta } \\
\text { directiva }\end{array}$ & Positivo & Acero y Alcalde (2012) \\
\hline Indicadores ambientales & Cantidad de indicadores & Positivos & Moneva y Ortas (2009) \\
\hline Edad de la organización & $\begin{array}{l}\text { Número de años en fun- } \\
\text { cionamiento }\end{array}$ & $\begin{array}{l}\text { Diferente } \\
\text { de cero }\end{array}$ & Choi (1999) \\
\hline Tamaño & $\begin{array}{l}\text { Logaritmo natural del ac- } \\
\text { tivo total }\end{array}$ & Positivo & $\begin{array}{l}\text { Preston y O'Bannon } \\
\text { (1997), Alonso J. et al. } \\
\text { (2016) }\end{array}$ \\
\hline Nivel de endeudamiento & $\begin{array}{l}\text { Pasivos totales/activos } \\
\text { totales. }\end{array}$ & $\begin{array}{l}\text { Diferente } \\
\text { de cero }\end{array}$ & Cuervo y Rivero (1986) \\
\hline
\end{tabular}

Fuente: elaboración propia.

Tras definir la variable dependiente y seleccionar los posibles factores que ayuden a explicar el desempeño financiero, se establece el siguiente modelo:

$$
\mathrm{QTOBIN}_{j t}=\beta_{0}+\beta_{1} \mathrm{M}_{j t}+\beta_{2} \mathrm{RA}_{j t}+\beta_{3} \mathrm{MI}_{j t}+\beta_{4} \mathrm{IA}_{j t}+\beta_{5} \mathrm{E}_{j t}+\beta_{6} \mathrm{~T}_{j t}+\beta_{7} \mathrm{NE}_{j t}
$$

Donde:

$\mathrm{M}_{j t}=$ Igualdad

$\mathrm{RA}_{j t}=$ Aseguramiento del reporte

$\mathrm{MI}_{j t}=$ Cantidad de miembros independientes

$\mathrm{IA}_{j t}=$ Cantidad de indicadores ambientales

$E_{j t}=$ Antigüedad de la compañía

$\mathrm{T}_{j \mathrm{t}}=$ Tamaño

$\mathrm{NE}_{j t}=$ Nivel de endeudamiento 


\section{ALGUNOS RESULTADOS OBTENIDOS}

Se obtuvo la matriz de correlación con el objetivo de observar las correlaciones existentes entre las variables estudiadas. En la tabla 4 se presentan las correlaciones y algunas estadísticas descriptivas de las variables.

Tabla 4. Estadísticas descriptivas y matriz de correlaciones

\begin{tabular}{|c|c|c|c|c|c|c|c|c|c|c|c|c|}
\hline & \multicolumn{4}{|c|}{ Estadísticas descriptivas } & \multicolumn{8}{|c|}{ Correlaciones } \\
\hline & Media & Desviación & Mínimo & Máximo & QTOBIN & M & RA & MI & IA & E & $\mathrm{T}$ & NE \\
\hline QTOBIN & 1,56 & 0,99 & 0,4 & 4,44 & 1 & & & & & & & \\
\hline M & 4.336 & 10.904 & 41 & 50.540 & 0,05 & 1 & & & & & & \\
\hline RA & 0,20 & 0,40 & 0 & 1 & 0,19 & 0,18 & 1 & & & & & \\
\hline MI & 2,53 & 2,79 & 0 & 9 & 0,02 & $-0,07$ & 0,41 & 1 & & & & \\
\hline IA & 12,84 & 10,36 & 0 & 48 & 0,4 & 0,24 & 0,35 & 0,05 & 1 & & & \\
\hline E & 49,89 & 30,77 & 3 & 131 & $-0,15$ & 0,2 & 0,06 & $-0,07$ & 0,12 & 1 & & \\
\hline $\mathrm{T}$ & 21,13 & 3,16 & 3 & 25,68 & 0,05 & $-0,04$ & $-0,3$ & $-0,31$ & $-0,29$ & 0,12 & 1 & \\
\hline $\mathrm{NE}$ & 0,37 & 0,24 & 0,02 & 1,57 & $-0,1$ & $-0,16$ & $-0,12$ & 0,26 & $-0,3$ & $-0,28$ & 0,07 & 1 \\
\hline
\end{tabular}

Fuente: resultados obtenidos del software $\mathrm{R}$.

Cabe destacar que la variable de los indicadores ambientales tiene la mayor correlación positiva con la variable Q de Tobin -estudios como el de Moneva y Ortas (2009) muestran tal relación-; también se evidencia una relación positiva entre el tamaño y la $\mathrm{Q}$ de Tobin. Por otro lado, se observa una correlación negativa entre la variable antigüedad y la $\mathrm{O}$ de Tobin, lo que indica que no en todos los casos la rentabilidad aumenta a la par con el número de años (Pozniak et al., 2011).

No obstante, para conocer el efecto conjunto y la significancia que tienen las variables independientes en la Q de Tobin se desarrolló un modelo de datos de panel, cuyos resultados se muestran en la tabla 5 .

Al estimar el modelo se obtuvo como resultado que, desde el punto de vista estadístico, tres variables tienen un efecto significativo en el desempeño financiero.

La cantidad de indicadores ambientales presenta un efecto positivo en el desempeño financiero $(\beta=0,039836, p<0,01)$; es decir, conforme las empresas sean más responsables con el ambiente y lo presenten en sus reportes de sostenibilidad a 
través de indicadores ambientales, obtendrán un mejor desempeño financiero. Lo anterior guarda coherencia con lo establecido por Moneva y Ortas (2009), ya que la información relacionada con el tema ambiental se erige cada vez más como un referente principal en la presentación dentro del GRI.

Tabla 5. Resultados del modelo datos de panel

\begin{tabular}{|c|c|c|c|c|c|}
\hline & Coeficiente & Error estándar & Valor $t$ & $\operatorname{Pr}(>|t|)$ & Significancia \\
\hline (Intercept) & $-0,284880$ & 0,836070 & $-0,340700$ & 0,734135 & \\
\hline M & 0,000000 & 0,000007 & 0,068500 & 0,945548 & \\
\hline RA & 0,099094 & 0,310620 & 0,319000 & 0,750488 & \\
\hline MI & 0,007381 & 0,043459 & 0,169800 & 0,865537 & \\
\hline IA & 0,039836 & 0,011777 & 3,382400 & 0,001083 & $* *$ \\
\hline E & $-0,008057$ & 0,004176 & $-1,929100$ & 0,057014 & $*$ \\
\hline T & 0,084669 & 0,034309 & 2,467800 & 0,015575 & $*$ \\
\hline NE & $-0,257590$ & 0,473240 & $-0,544300$ & 0,587635 & \\
\hline
\end{tabular}

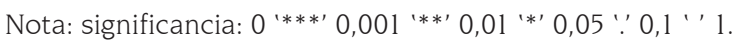

Fuente: elaboración propia con resultados obtenidos del software R.

Tal como se pronosticaba en función de la revisión teórica, el tamaño de la empresa arrojó un efecto positivo sobre el desempeño financiero $(\beta=0,084669$, $\mathrm{p}<0,05$ ). A mayor tamaño (representado en los activos) tenga la empresa, mayor va a ser la contribución de este a su desempeño financiero. Esto reafirma lo expuesto por Preston y O'Bannon (1997): cuando la empresa tiene buenos activos dispone de más recursos, y esto se ve reflejado en el desempeño financiero.

La antigüedad, por su parte, tiene un efecto negativo en el desempeño financiero $(\beta=-0,008057, p<0,1)$ : esto es, cuantos más años tenga una empresa, menor será su incidencia en el desempeño financiero (Pozniak et al., 2011). Según Moreno, García, y Pablo (2003), lo anterior puede explicarse por cuanto, a medida que pasan los años, las empresas dejan de crecer debido a que llegan a un estado de madurez, en el cual deben generar cada vez mejores innovaciones para seguir en la senda del crecimiento.

Las variables de cantidad de mujeres, aseguramiento de los reportes, miembros independientes en la junta directiva y el nivel de endeudamiento carecen de capa- 
cidad para dar explicación al desempeño financiero en las empresas colombianas para el periodo estudiado. Por lo tanto, no se puede asegurar que tengan un efecto positivo o negativo sobre la $\mathrm{Q}$ de Tobin, ya que no presentaron un nivel de confianza significativo en términos estadísticos.

Con los resultados observados en la tabla 5 se observa que la información no financiera que se reporta en los informes de sostenibilidad tiene impactos sobre el desempeño financiero de las firmas. Los indicadores ambientales, que se obtienen de los informes de sostenibilidad de las empresas, tienen la capacidad de influir positivamente en el desempeño financiero, y los convierten en una variable que las compañías deben gestionar para maximizar el valor financiero.

Como lo señala Choi (1999), el impacto positivo de los indicadores ambientales en el desempeño financiero se puede explicar debido a que los grupos de interés, tales como clientes, proveedores, empleados y demás acreedores, prefieren las empresas social y ambientalmente responsables; ello hace que estas compañías generen mayores ingresos, cuenten con mejor calidad en sus materias primas y tengan una mejor base de empleados.

\section{CONCLUSIONES}

El interés por reportar información no financiera ha ido en aumento, y el GRI se presenta como alternativa para hacerlo. Las empresas tienen la oportunidad de reportar indicadores útiles para medir sus prácticas socioambientales; esto da muestra de confiabilidad y de transparencia, contribuye a la generación de valor agregado y se convierte en parte de ventaja competitiva en el mercado.

Según Fifka (2013), la RSE se ha vinculado al desempeño financiero de las compañías desde mediados de la década de 1970, cuando comenzaron a realizarse estudios de tipo empírico con el fin de identificar la relación existente entre la responsabilidad social empresarial y el desempeño financiero. Sin embargo, como lo señala Borjas (2007), no se ha logrado un conceso en los resultados, los cuales no han sido homogéneos ni tampoco esclarecedores.

Del estudio descrito en este artículo, con 30 empresas colombianas y 94 observaciones empresa-año que reportaron información bajo el GRI durante el periodo 2012-2015, se concluye que los sectores en que más se aplica el GRI en Colombia son energético $(24,5 \%)$, minero (14,9\%), industrial $(14,9 \%)$, de telecomunicaciones $(12,8 \%)$ y comercial $(12,8 \%)$, los cuales tienen alto impacto social y ambiental. Estos resultados concuerdan con lo expuesto por Almeida, Marimon y Llach (2015): estos autores señalan que las empresas que tienen un alto impacto social y am- 
biental derivado de sus operaciones, hacen mayor uso de los reportes de RSE para generar confianza con los grupos de interés.

La antigüedad de la empresa, su tamaño y la cantidad de indicadores ambientales presentes en los informes de sostenibilidad se identifican como variables que inciden en el desempeño financiero de las empresas que los reportan. Tal como se pronosticaba, el tamaño de la empresa y la cantidad de indicadores ambientales tienen un efecto positivo en el desempeño financiero, contrario lo que sucede con la antigüedad de las firmas. No sucede lo mismo con el nivel de endeudamiento, los miembros independientes, la cantidad de mujeres y el aseguramiento de los reportes de sostenibilidad, que no tienen un efecto estadístico significativo sobre la $\mathrm{Q}$ de Tobin.

Los indicadores ambientales juegan un papel fundamental en los reportes de sostenibilidad, ya que son un referente principal dentro del GRI que afecta de forma positiva el desempeño financiero de la firma (Moneva y Ortas, 2009).

Debido a que la elaboración y presentación de los reportes de sostenibilidad es voluntaria, el acceso a esa información es limitado. Esto lleva a que las empresas tengan la posibilidad de no presentarlos de manera continua durante todos los años, y a no contar con una fecha específica de presentación.

\section{REFERENCIAS}

Acero, I. y Alcalde, N. (2012). Gobierno corporativo y rendición de cuentas: ¿existe algún efecto sobre la performance empresarial? En: Revista de Contabilidad, Vol. 15, n. ${ }^{\circ}$ 1, p. 143-178. DOI: 10.1016/ S1138-4891(12)70041-9.

Alonso-Almeida, M. M.; Rodríguez, M. P; Cortez, K. y Abreu, J. L. (2012). La responsabilidad social corporativa y el desempeño financiero: un análisis en empresas mexicanas que cotizan en bolsa. En: Contaduría y Administración, Vol. 57, n. 1, enero-marzo, p. 53-77.

Alonso, J.; Saraite, L.; Haro de Rosario, A. y Caba Pérez, C. (2016). Sector bancario a nivel mundial y los factores que influyen en su información sobre responsabilidad social corporativa. En: Revista Contaduría Universidad de Antioquia, n. ${ }^{\circ}$ 68, p. 213-233.

Blazenko, G. W. (1987). Managerial Preference, Asymmetric Information, and Financial structure. En: The journal of finance, Vol. 42, n. ${ }^{\circ}$, p. p39-862.

Borjas, C. (2007). Responsabilidad Social Empresarial y Desempeño Financiero: Un Enfoque Estratégico. En: Revista de Ciencias Empresariales y Economía, n. ${ }^{\circ}$ 6, p. 137-146.

Callan, S. y Thomas, J. (2009). Corporate Financial Performance and Corporate Social Performance: An Update and Reinvestigation. En: Corporate Social Responsibility and Environmental Management, Vol. 16, n. ${ }^{\circ}$, p. $61-78$. 
Choi, J. (1999). An Investigation of The Initial Voluntary Environmental Disclosures Made In Korean Semi-Annual Financial Reports. En: Pacific Accounting Review, Vol. 11, n. ${ }^{\circ}$ 1, p. 73-102.

Correa, J. (2007). Evolución histórica de los conceptos de responsabilidad social empresarial y balance social. En: Semestre Económico, Vol. 10, n. ${ }^{\circ}$ 20, julio-diciembre, p. 87-102.

Cuervo, A. y Rivero, P. (1986). El análisis económico-financiero de la empresa. En: Revista Española de Financiación y Contabilidad, Vol. XVI, n. ${ }^{\circ}$ 49, p. 15-33.

Duque, Y.; Cardona, M. y Rendón, J. (2013). Responsabilidad Social Empresarial: Teorías, índices, estándares y certificaciones. En: Cuadernos de Administración, Vol. 29, n. . 50, julio-diciembre, p. 196-206.

El Ghoul, S.; Ghedami, O.; Kwok, C. y Mishra, D. (2011). Does corporate social responsibility affect the cost of capital? En: Journal of Banking and Finance, Vol. 35, n. ${ }^{\circ}$ 9, p. 2388-2406.

Fatemi, A.; Fooladi, I. y Tehranian, H. (2015). Valuation effects of corporate social responsibility. En: Journal of Banking \& Finance, Vol. 59, p. 182-192. DOI: 10.1016/j.jbankfin.2015.04.028.

Ferrero, Y. (2013). Consecuencias de las prácticas de sostenibilidad en el coste de capital y en la reputación corporativa. En: Spanish Accounting Review, Vol. 17, n. 2, p. $153-162$. DOI: 10.1016/j. rcsar.2013.08.008

Fifka, M. (2013). Corporate responsibility reporting and its determinants in comparative perspective-a review of the empirical literature and a meta-analysis. En: Business Strategy and the Environment, Vol. 22, n. ${ }^{\circ} 1$, p. $1-35$.

Garay, U. y González, M. (2005). CEO and Director Turnover in Venezuela. IDB Working Paper, n. $212,48 \mathrm{p}$.

García, A.; Zamora y Ruiz, M. (2009). Una aproximación a las relaciones de gobierno corporativo en las empresas españolas, 23p.

Godoy, L. y Mladinic, A. (2009). Estereotipos y Roles de Género en la Evaluación Laboral y Personal de Hombres y Mujeres en Cargos de Dirección. En: PSYKHE, Vol. 18, n. 2, p. 51-64.

Gómez, M. y Quintanilla, D. (2012). Los informes de responsabilidad social empresarial: su evolución $y$ tendencias en el contexto internacional y colombiano. En: Revistas Javeriana, Vol. 13, n. ${ }^{\circ} 32$, enero-junio, p. $121-158$.

Hamid, F. (2004). Corporate Social Disclose by Banks and Finance Companies: Malaysian Evidence. En: Corporate Ownership and Control, Vol. 1, n. ${ }^{\circ}$ 4, p. 118-130.

Kim, Y.; Li, H. y Li, S. (2014). Corporative Social Responsibility and Stock Price Crash Risk. En: Journal of Banking \& Finance, Vol. 43, p. 1-13.

Luo, X. y Bhattacharya, C. (2006). Corporate Social Responsibility, Customer Satisfaction, and Market Value. En: Journal of Marketing, Vol. 21, n. ${ }^{\circ}$ 1, p. 39-53.

Marimon, F; Alonso, M; Rodríguez, M. y Cortez, K. (2012). The Worldwide Diffusion of the Global Reporting Initiative: What is the Point? En: Journal of Cleaner Production. Vol. 33, septiembre, p. 132-144. DOI: 10.1016/j.jclepro.2012.04.017.

Moneva, J. y Ortas, E. (2009). Desarrollo sostenible e información corporativa. En: Economía Industrial, n. ${ }^{\circ}$ 371 (Edición especial: Industria y medio ambiente: el reto de la sostenibilidad), p. 139-154. 
La información no financiera y el desempeño financiero empresarial

Montoro, C. y Navarro, A. (2010). Estimación de la Q de Tobin para la economía peruana. En: Revista Estudios Económicos, Vol. 19, septiembre, p. 33-45.

Moreno, J.; García, A. y Pablo, F. (2003). Análisis de la relación entre el crecimiento empresarial, la edad de la empresa y la estructura de propiedad. En: Universidad de Alcalá, n. 3, p. 1-41.

Nelling, E. y Webb, E. (2009). Corporate Social Responsibility and Financial Performance: The "Virtuous Circle" Revisited. En: Review of Quantitative Finance and Accounting, Vol. 32, n. ${ }^{\circ}$ 2, febrero, p. 197-209.

Núñez, G. (2003). La responsabilidad social corporativa en un marco de desarrollo sostenible. Santiago de Chile: Cepal, 70p.

Pérez, J. A. (2001). El papel de la mujer en la empresa. España, 16p.

Plumlee, M.; Brown, D.; Hayes, R. M. y R.S, y M. (2015). Voluntary Environmental Disclosure Quality and Firm Value: Further Evidence. En: Journal of accounting and public policy, Vol. 34, n. ${ }^{\circ}$, julio-agosto, p. 336-361.

Pozniak, L.; Ferauge, P.; Arnnone, L. y Geerts, A. (2011). Determinants of Internet Corporate Social Responsibility Communication. En: Global Journal of Business Research, Vol. 5, n. ${ }^{\circ}$ 4, julio, p. 1- 14.

Preston, L. y O'Bannon, D. (1997). The Corporate Social-Financial Performance Relationship. En: Business and Society, Vol. 36, n. ${ }^{\circ}$, p. 419-429.

Rodríguez, L. y Ríos, L. (2016). Evaluación de Sostenibilidad con Metodología GRI. En: Dimensión Empresarial, Vol. 14, n. ${ }^{\circ}$ 2, p. 73-89.

Sáenz, A.; Haroa, A.; Caba, M. y Benítez, M. (2014). Determinantes de la información sostenible divulgada por empresas de electricidad: Un estudio internacional. En: Revista Retos, Vol. 7, n. ${ }^{\circ}$ 1, p. 15-28.

Viteri, J. (2010). Responsabilidad Social. En: Enfoque, n. ${ }^{\circ}$ 1, Universidad Tecnológica Equinoccial, p. $90-100$.

Wang, H. y Qian, C. (2011). Corporate Philanthropy and Corporate Financial Performance: The Roles of Stakeholder Response and Political Access. En: Academy of Management, Vol. 54, n. ${ }^{\circ}$ 6, p. 1159-1181.

Willis, A. (2003). The Role of the Global Reporting Initiative's Sustainability Reporting Guidelines in the Social Screening of Investments. En: Journal of Business Ethics, Vol. 43, n. ${ }^{\circ}$ 3, marzo, p. 233-237. 
ANEXOS

ANEXO 1. EMPRESAS INCLUIDAS EN EL ESTUDIO

\begin{tabular}{|c|c|}
\hline Número & Empresa \\
\hline 1 & Avianca Holdings S.A. \\
\hline 2 & Celsia \\
\hline 3 & Cementos Argos \\
\hline 4 & Ecopetrol \\
\hline 5 & EEB \\
\hline 6 & Endesa Colombia \\
\hline 7 & ETB \\
\hline 8 & Gas Natural Colombia \\
\hline 9 & Grupo Éxito \\
\hline 10 & Holcim Colombia \\
\hline 11 & ISA \\
\hline 12 & Isagén \\
\hline 13 & Mineros S.A \\
\hline 14 & Pacific Exploration \& Production \\
\hline 15 & Smurfit Kappa Cartón de Colombia \\
\hline 16 & Telefónica Colombia \\
\hline 17 & UNE \\
\hline 18 & Biomax \\
\hline 19 & Clínica Universidad de La Sabana \\
\hline 20 & Construcciones El Cóndor \\
\hline 21 & Promigás \\
\hline 22 & Zona Franca de Barranquilla \\
\hline 23 & Enka \\
\hline 24 & Grupo Argos \\
\hline 25 & Corferias \\
\hline 26 & Expreso Brasilia \\
\hline 27 & Grupo Familia \\
\hline 28 & Acesco \\
\hline 29 & Canacol Energy \\
\hline 30 & Continental Gold \\
\hline
\end{tabular}

